\title{
EFFECTIVE AGEING LINEAR VISCOELASTIC PROPERTIES OF COMPOSITES WITH PHASE PRECIPITATION: COMPARISONS BETWEEN NUMERICAL AND ANAL YTICAL HOMOGENIZATION APPROACHES
}

\author{
TULIO HONORIO ${ }^{*}$, BENOIT BARY ${ }^{\dagger}$ AND JULIEN SANAHUJA ${ }^{\dagger \dagger}$ \\ CEA, DEN, DPC, SECR, Laboratoire d'Etude du Comportement des Bétons et des Argiles \\ F-91191 Gif-sur-Yvette, France \\ e-mail: tulio.honorio-de-faria@enpc.fr \\ ${ }^{\dagger}$ CEA, DEN, DPC, SECR, Laboratoire d'Etude du Comportement des Bétons et des Argiles \\ F-91191 Gif-sur-Yvette, France \\ e-mail: benoit.bary@cea.fr \\ ${ }^{\dagger \dagger}$ Département Mécanique des Matériaux et des Composants, EDF R\&D, \\ Site des Renardières, Avenue des Renardières, 77818 Moret-Sur-Loing Cedex, France \\ e-mail: julien.sanahuja@edf.fr
}

Key words: Linear Viscoelasticity, Ageing, 3D Simulations, Heterogeneous materials, Homogenization.

\begin{abstract}
Dissolution and precipitation processes are present in key phenomena affecting the behavior of cement-based materials. Additionally, cement-based materials exhibit viscoelastic behavior. Recently, analytical homogenization tools have been developed to upscale the effective properties of composites in an ageing linear viscoelastic framework [1,2]. Taking advantage of these tools, an extension of Bazant's original solidification theory [3] was proposed in a 3D tensorial context [4]. In this paper, we propose to benchmark these analytical approaches by comparing with numerical homogenization to estimate the behavior of ageing composites in different scenarios. To this end, 3D numerical samples are generated by randomly distributing inclusions of various sizes and shapes in a box [5,6]. We compare the response of solidification in two main morphologies: spherical and convex polyhedral inclusions. The results provided here go towards a better description of the dissolution/precipitation processes, which is an important feature in the characterization of cement-based materials ageing behavior.
\end{abstract}

\section{INTRODUCTION}

Dissolution and precipitation processes are present in key phenomena affecting the behavior of cement-based materials. At earlyage, due to hydration process, new phases are formed within capillary pores; at late ages, the material can be submitted to degradation processes in which hydrated phases dissolve. These processes reflect in an ageing behavior of the material.

Moreover, cement-based materials, as other geomaterials, exhibit viscoelastic behavior. Recently, analytical homogenization tools have been developed to upscale the effective 
properties of composites in an ageing linear viscoelastic framework $[1,2]$.

Bazant [7] showed that an ageing effective behavior can be observed in materials in which one of the constituents shows a change in its volume fraction in time, as occurs for example in a solidification process. This effect is observed even if the basic constituents are non-ageing per se [8]. An extension of this idea to a 3D tensorial context was carried out by Sanahuja [4]. The results showed that the topology of dissolution/precipitations affected the overall response of the material $[9,10]$.

In this paper, we propose to benchmark these analytical approaches by comparing with numerical homogenization to estimate the behavior of ageing composites in different scenarios. To this end, 3D numerical samples are generated by randomly distributing inclusions of various sizes and shapes in a box $[5,6]$. We compare the response of solidification in two main morphologies: spherical and convex polyhedral inclusions.

\section{REPRESENTATION OF DISSOLUTION AND PRECIPITATION PROCESSES}

Analytical and numerical strategies to cope with the representation of dissolution/ precipitation are presented in the following. Hereafter, we assume that precipitation occurs in pores. Extensions to cases with dissolution or combined one step dissolution/precipitation are straightforward.

\subsection{Analytical framework}

Defining the product operator $(f \circ$ $g)\left(t, t_{0}\right) \equiv \int_{t^{\prime}=-\infty}^{t} f\left(t, t^{\prime}\right) d_{t^{\prime}} g\left(t^{\prime}, t_{0}\right), \quad$ the stresses $\sigma$ can be then written in terms of strains $\varepsilon$ and the relaxation $\mathbb{R}$ 4-rank tensor as $[2,11]$ :

$$
\sigma(x, t)=\mathbb{R}(x, t, .) \stackrel{\circ}{\stackrel{m}{:}} \varepsilon(x, .)
$$

With this correspondence principle, analytical homogenization schemes can be derived in ageing linear viscoelasticity (e.g. for schemes based on Eshelby solution [2] or in specific morphologies [1]). Hereafter, we consider Mori-Tanaka estimations for isotropic spherical inclusions following Sanahuja [2]. The integrals are numerically evaluated by a trapezoidal rule (see [2] for details).

To represent solidification, we use the extensions of solidification theory proposed by Sanahuja [4]. The changes in the volume fraction of phases are taken into account with analytical homogenization replacing the precipitating phase by fictitious ageing phases. The effective behavior is then estimated through non ageing linear viscoelastic homogenization. In this context, the relaxation tensor for a given phase solidifying at time $t_{i}^{S}$ is identified by:

$$
\mathbb{R}_{\mathrm{i}}\left(\mathrm{t}, \mathrm{t}_{0}\right)=\mathbb{R}_{\mathrm{s}}\left(\mathrm{t}-\mathrm{t}_{0}\right) \mathrm{H}\left(\mathrm{t}_{0}-\mathrm{t}_{\mathrm{i}}^{\mathrm{s}}\right)
$$

where $\mathbb{R}_{s}\left(t-t_{0}\right)$ is the relaxation tensor of the solidified material and $t_{0}$ is the age of loading.

This strategy involves a discretization of the ageing function in $\mathrm{n}$ steps. Here, as in [4], the solidification times are chosen so that the same volume fraction of solid $\mathrm{f}_{\text {age }}^{\infty} / \mathrm{n}$ precipitates at each $t_{i}^{s}$. Correspondingly, we have [4]:

$$
f_{\text {age }}\left(t_{i}^{s}\right)=\left(i-\frac{1}{2}\right) \frac{f_{a g e}^{\infty}}{n}
$$

with $f_{\text {age }}^{\infty}$ being the final value of the volume fraction of the phase solidifying. The estimation of the effective properties then is made by determining the properties of a fictitious n-phases composite. Sanahuja [4] showed that the precipitation mechanism and the kinetics of the ageing process affect directly the effective ageing viscoelastic response.

Figure 1 shows a scheme of the solidification processes as accounted for analytically here for a composite with matrix/inclusions morphology (so that MoriTanaka can be applied).

\subsection{Numerical framework}

Two numerical samples (Figure 2) are studied: a) with a volume fraction of $30 \%$ of 
spherical inclusions and (b) with $40 \%$ of convex polyhedral inclusions obtained from Voronoi decomposition [12]. These numerical samples are generated by randomly distributing the inclusions in a box. The procedure for constructing the mesostructures is detailed in [13] and [14]. The open-source python library Combs based on the ComputerAided Design code Salome (www.salomeplatform.org) is used to generate geometry and meshes of the mesostructures [13]. The GJK 3D algorithm has been recently implemented in Combs for fast convex particles distance computation (see e.g. http://www.dyn4j.org/ 2010/04/gjkdistance-closest-points). The corresponding meshes are automatically generated with meshing software directly plugged in Salome. The number of tetrahedral elements is 1.75 and 2.37 millions for the microstructures of Figure 2 a) and b), respectively. Static uniform boundary conditions (SUBC) are generally adopted (otherwise stated), since they are reported to return more adequate results for composites in which the inclusions are stiffer than the matrix $[1,15]$ than kinematic uniform boundary conditions.

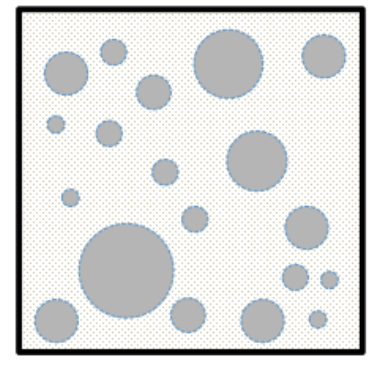

$$
\mathrm{t}=\mathrm{t}_{\mathrm{s} 0}
$$

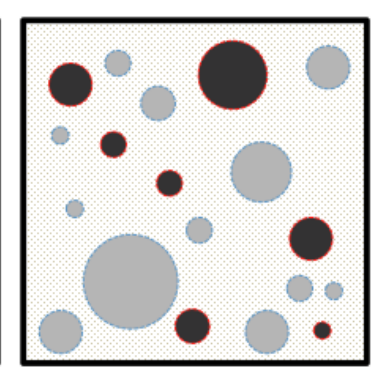

$$
\mathrm{t}=\mathrm{t}_{1}^{\mathrm{s}}>\mathrm{t}_{0}^{\mathrm{s}}
$$
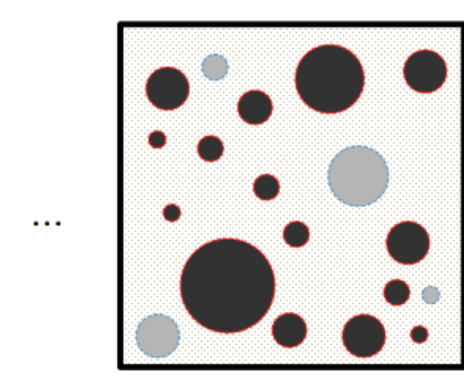

Precipitating phase

Pores

$$
\mathrm{t}=\mathrm{t}_{\mathrm{n}}^{\mathrm{s}}>\mathrm{t}^{\mathrm{s}}
$$

Figure 1: Representation of solidification processes: matrix/inclusions configuration with massive precipitation in pores.

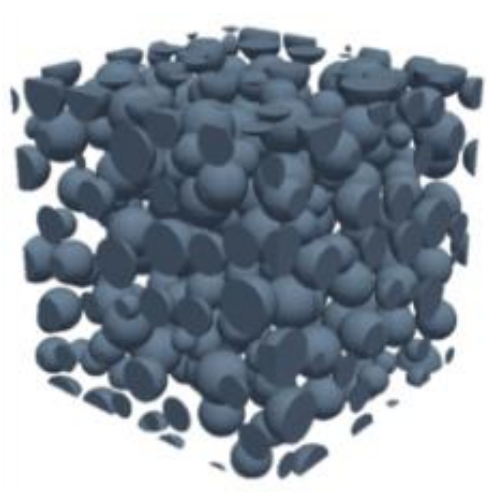

(a)

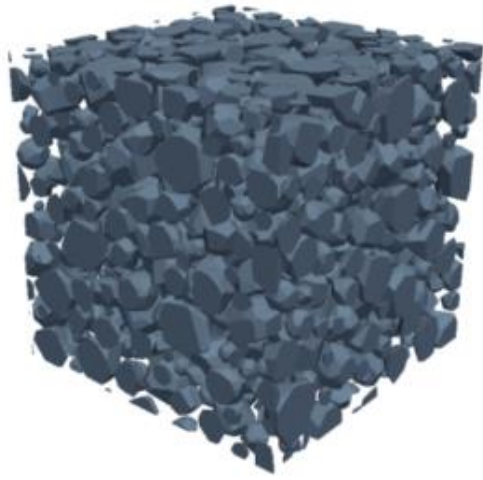

(b)

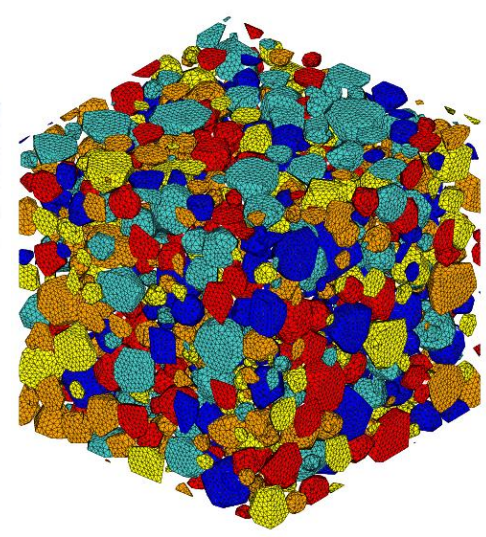

(c)

Figure 2: Numerical samples: $30 \%$ of spherical inclusions (a), $40 \%$ of convex polyhedral inclusions (b). Representation of solidification process with a discretization of $n d=5$ timesteps (c).

The simulations are performed with the finite element code Cast3M (www.cast3m.fr), in which the ageing viscoelastic constitutive behavior is implemented following an algorithm similar to the one of [16], by means of the MFront code generator (http://tfel. sourceforge.net/, [17]). The solidification process is modelled by decomposing randomly 
the different inclusions in as many sub-phases as necessary to comply with the required discretization in terms of sub-volume. As this decomposition is random, a great number of realizations can be generated. An example is shown on Figure 2(c) in the case of the $40 \%$ polyhedral inclusions and a discretization of aggregates in 5 sub-volumes.

\section{RESULTS}

We consider a porous composite material in which the material constituting the matrix phase precipitates within the pores (massive precipitation [4]) following an ageing function.

Two ageing functions are studied, a sigmoid and a linear one, as shown in Figure 3 , given respectively by:

$$
\begin{gathered}
f_{a g e}^{A}(t)=\frac{t^{4}}{1+t^{4}} f_{a g e}^{\infty} \\
f_{a g e}^{B}(t)=\min \left(1, \frac{1}{3} t\right) f_{a g e}^{\infty}
\end{gathered}
$$

The behavior of the matrix (and precipitating phase, which are the same here) is represented by a generalized Maxwell model:

$$
X\left(t-t^{\prime}\right)=x_{0}+\sum_{i} x_{i} \exp \left[-\left(t-t^{\prime}\right) / x_{i}\right.
$$

where $X$ is the shear or bulk relaxation functions, $\mu$ and $\mathrm{k}$, respectively. $\mathrm{x}_{\mathrm{i}}$ are the Maxwell generalized parameters associated to each relaxation function and $\chi_{i}$ is the characteristic time of each chain. Table 1 gathers the values of the parameters used in this paper. There, the values are normalized with respect to a chosen $\mu^{\prime}$ and $\mathrm{k}^{\prime}$.

Table 1: Properties of the matrix and precipitating phase based on Maxwell generalized behavior

\begin{tabular}{cccc}
\hline & $x_{0}$ & $x_{1}$ & $x_{2}$ \\
\hline$k / k^{\prime}$ & 1 & 1 & 1 \\
\hline$\mu / \mu^{\prime}$ & $1 / 2$ & $1 / 2$ & $1 / 2$ \\
\hline$\chi / \tau$ & - & 0.1 & 1 \\
\hline
\end{tabular}

The pores are treated as elastic phases with $\mu$ and $\mathrm{k}$ equal to 0 .

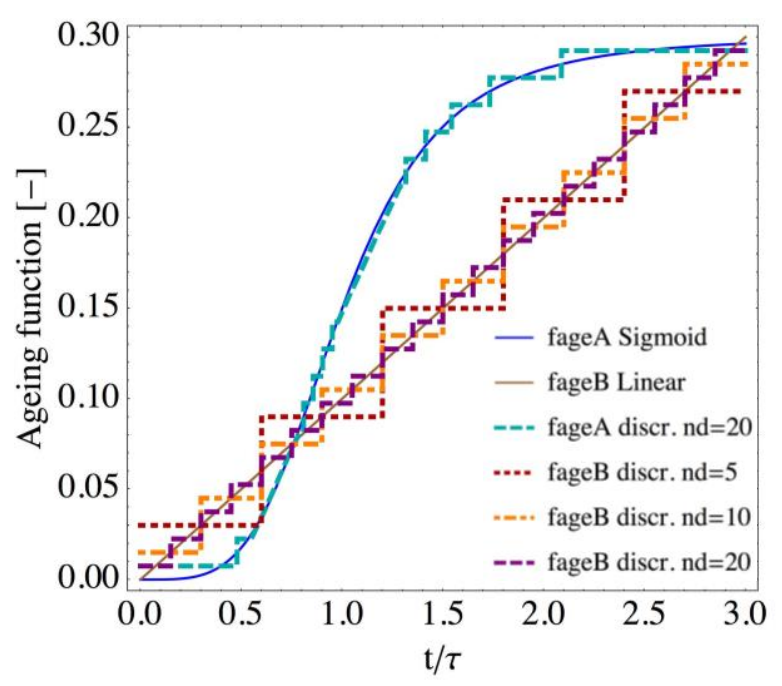

Figure 3: Ageing functions and discretization

\subsection{Spherical inclusions}

Figure 4 shows the components of relaxation tensor for both analytical and numerical estimations in the $30 \%$ spherical inclusions case. Recall that in the isotropic case we have:

$R_{1111}=R_{2222}=R_{3333}=k\left(t, t^{\prime}\right)+\frac{4}{3} \mu\left(t, t^{\prime}\right)$

$R_{1122}=R_{1133}=R_{2233}=k\left(t, t^{\prime}\right)-\frac{2}{3} \mu\left(t, t^{\prime}\right)$

$R_{1212}=R_{1313}=R_{2323}=\mu\left(t, t^{\prime}\right)$

The same solidification discretization was used in both cases, $n d=20$. The ageing function is linear $\left(f_{a g e}^{B}\right)$. In analytical estimations 200 timesteps are used. As already mentioned static uniform boundary conditions (SUBC) are used in numerical simulation. Numerical and analytical results are in fairly good agreement, especially with $\mathrm{R}_{1111}$ and $\mathrm{R}_{1212}$ components.

Note that, as expected, the effective behavior of the composite is ageing even if the behaviors of the constituent phases are nonageing (non-ageing linear viscoelastic for both matrix and precipitating phase, and "elastic" pores). Figure 5 shows the influence of solidification discretization ( $n d=5,10$ or 20 timesteps). The linear ageing function $\left(f_{a g e}^{B}\right)$ is used. We note that the analytical solutions are more sensible to solidification discretization than FEM solutions. 

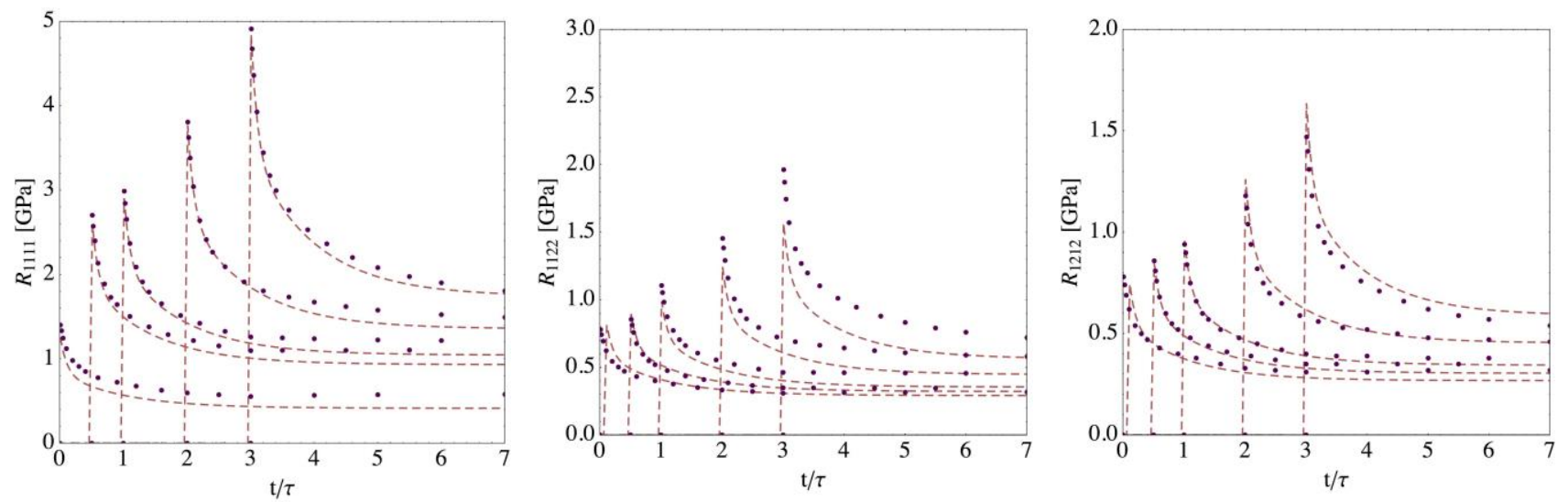

Figure 4: Components of relaxation tensor: comparison between analytical (full lines) and numerical results (dots) for the same solidification discretization. Volume fraction of inclusions is $30 \%$ and the ageing function is linear $\left(f_{a g e}^{B}\right)$.
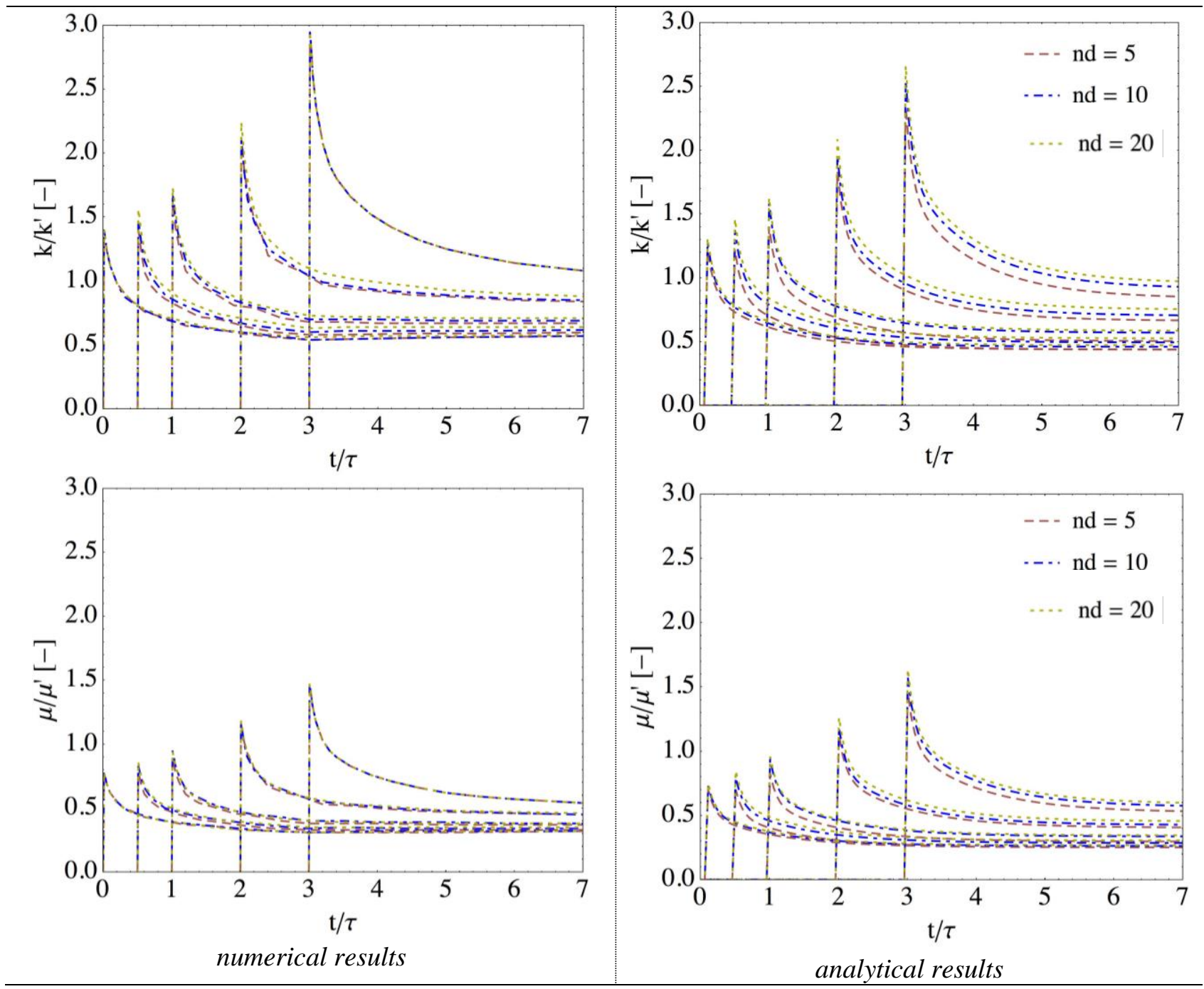

Figure 5: Influence of solidification discretization. Volume fraction of inclusions is $30 \%$ and the ageing function is linear $\left(f_{\text {age }}^{B}\right)$. 

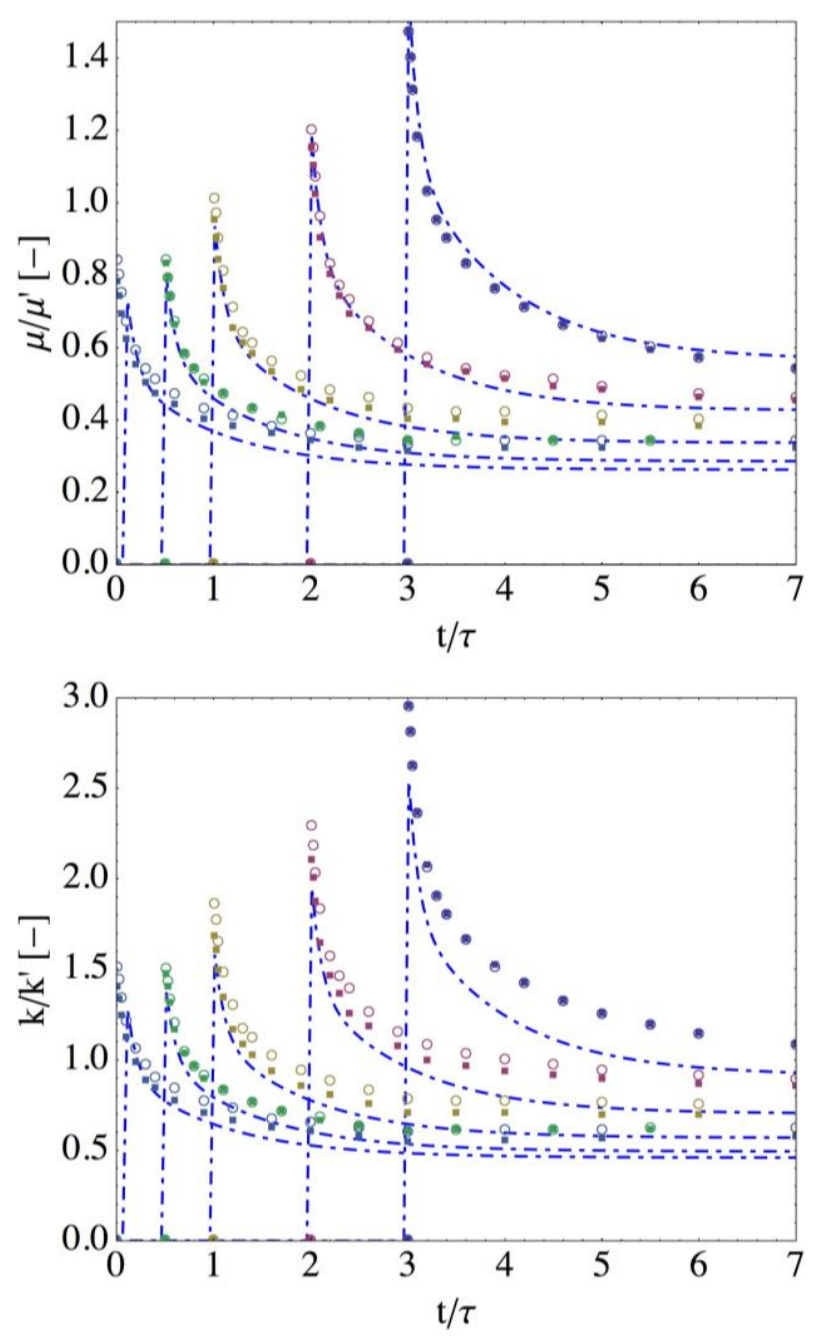

Figure 6: Influence of the boundary conditions. Numerical simulations are the dots (SUBC: full squares and KUBC: empty circles). Dot-Dashed curves are analytical results (as in Figure 5). 10 timesteps were used in the solidification discretization in all three cases. The ageing function is linear $\left(f_{\text {age }}^{B}\right)$.

Figure 6 shows results with SUBC and kinematic uniform (KUBC) boundary conditions piloted in stresses compared to analytical results in the case of the $30 \%$ volume fraction. Solidification was discretized in 10 timesteps in all cases. We observe that both boundary conditions returned close results.

Figure 7 shows the results with the sigmoidal ageing function $\left(f_{a g e}^{A}\right)$. A reasonable agreement is observed again between both estimations.
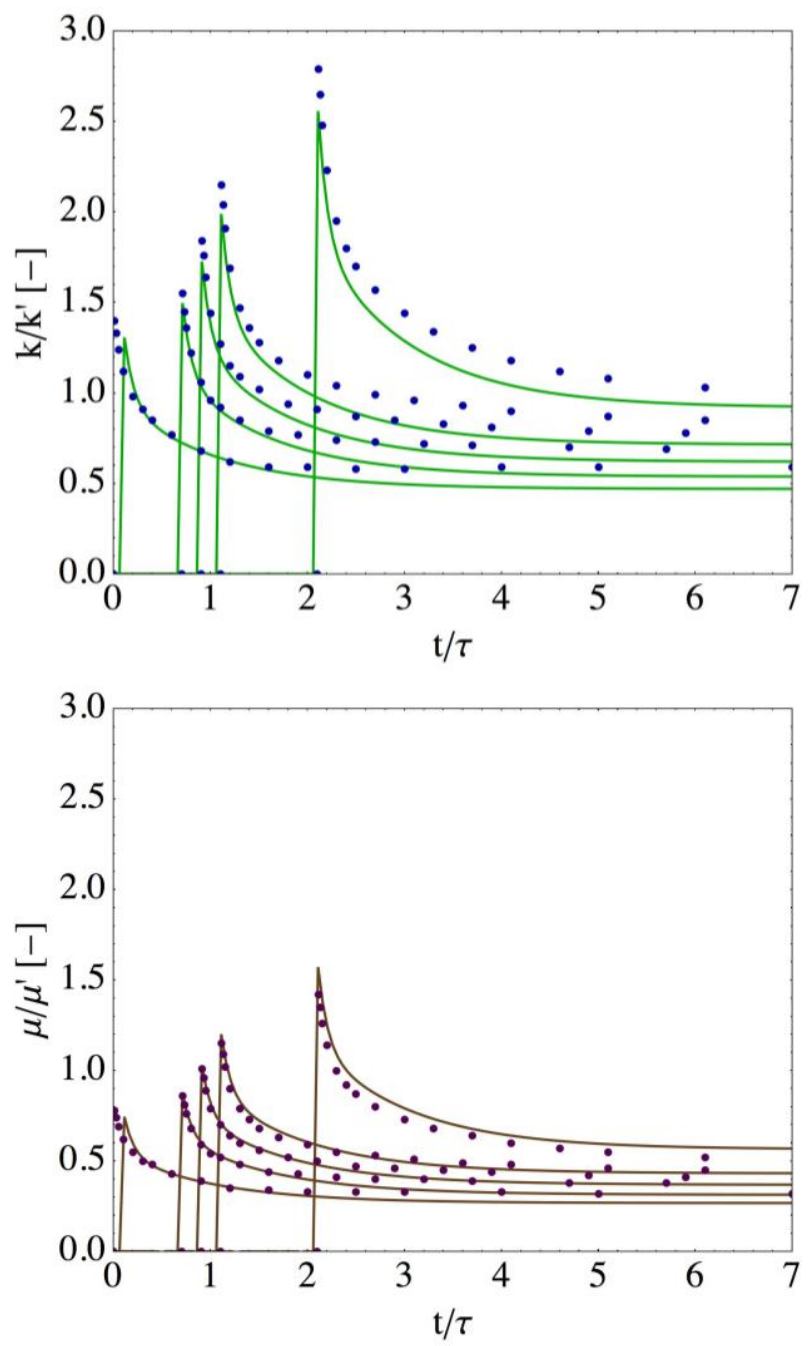

Figure 7: Bulk and shear relaxation functions: comparison between analytical (full lines) and numerical results (dots) for the same solidification discretization. Volume fraction of inclusions is $30 \%$ and the ageing function is sigmoidal $\left(f_{\text {age }}^{A}\right)$. SUBC was used in the numerical simulations.

Figure 8 shows the creep response (in terms of $J_{1111}$ component of creep tensor) for numerical and analytical results. Creep tensor can be obtained with analytical estimations by performing an inversion with respect to the integral operator. With numerical simulations, this component can be obtained directly from the macroscopic strains since a creep test with constant applied stresses is performed. It has been verified that the results are comparable to the analytical estimations $[1,18]$. We observe that the numerical results show a non 
monotonous evolution with some discontinuities in the derivatives for loading times $\mathrm{t}^{\prime}<2$ time units. This effect, which can be due to the discretization in time and space, would deserve further investigations.

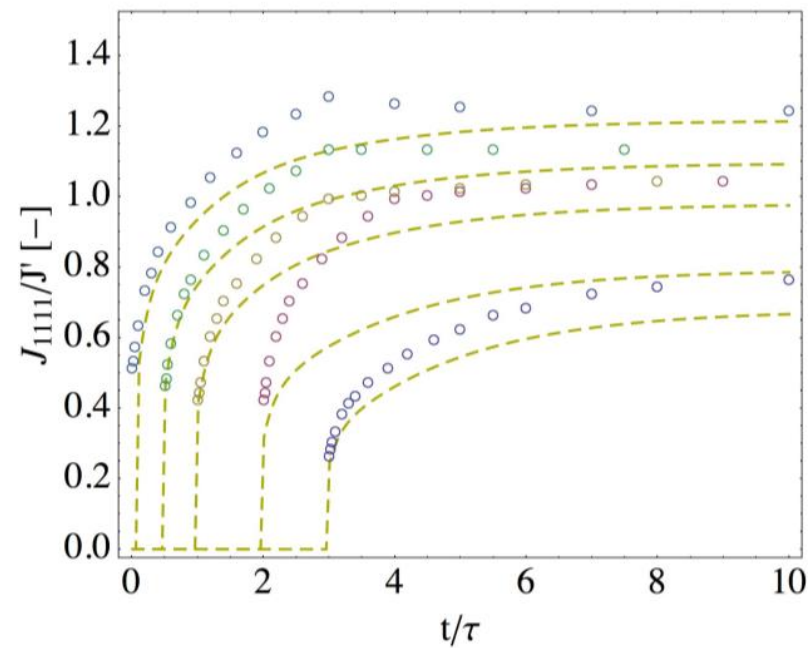

Figure 8: Creep response: $J_{1111}$ component of creep tensor. Dots are numerical results and dashed lines analytical results. Volume fraction of inclusions is $30 \%$ and the ageing function is linear $\left(f_{a g e}^{B}\right)$.

\subsection{Polyhedral inclusions}

Simulations with polyhedral inclusions were performed and compared to the analytical estimations. The results are shown in Figure 9. The volume fraction of the inclusions is $40 \%$. The linear ageing function is considered.

Again, an adequate agreement is observed between both analytical and numerical results. Slightly superior differences between the results are obtained when compared to the case with spherical inclusions. In addition to the effect of the shapes (i.e. polyhedral in the FE simulations and spherical in the analytical model), the effect of the relatively high volume fraction in MT estimates can also play a role in these differences.

\section{CONCLUSIONS}

A comparison between (semi-)analytical and numerical (FEM 3D simulations) estimations of the effective properties of materials with an ageing/solidifying linear viscoelastic behavior was performed. Both solutions returned results in fairly good agreement. As expected, the obtained effective behavior is ageing even if the behavior of the constituent phases is not ageing.
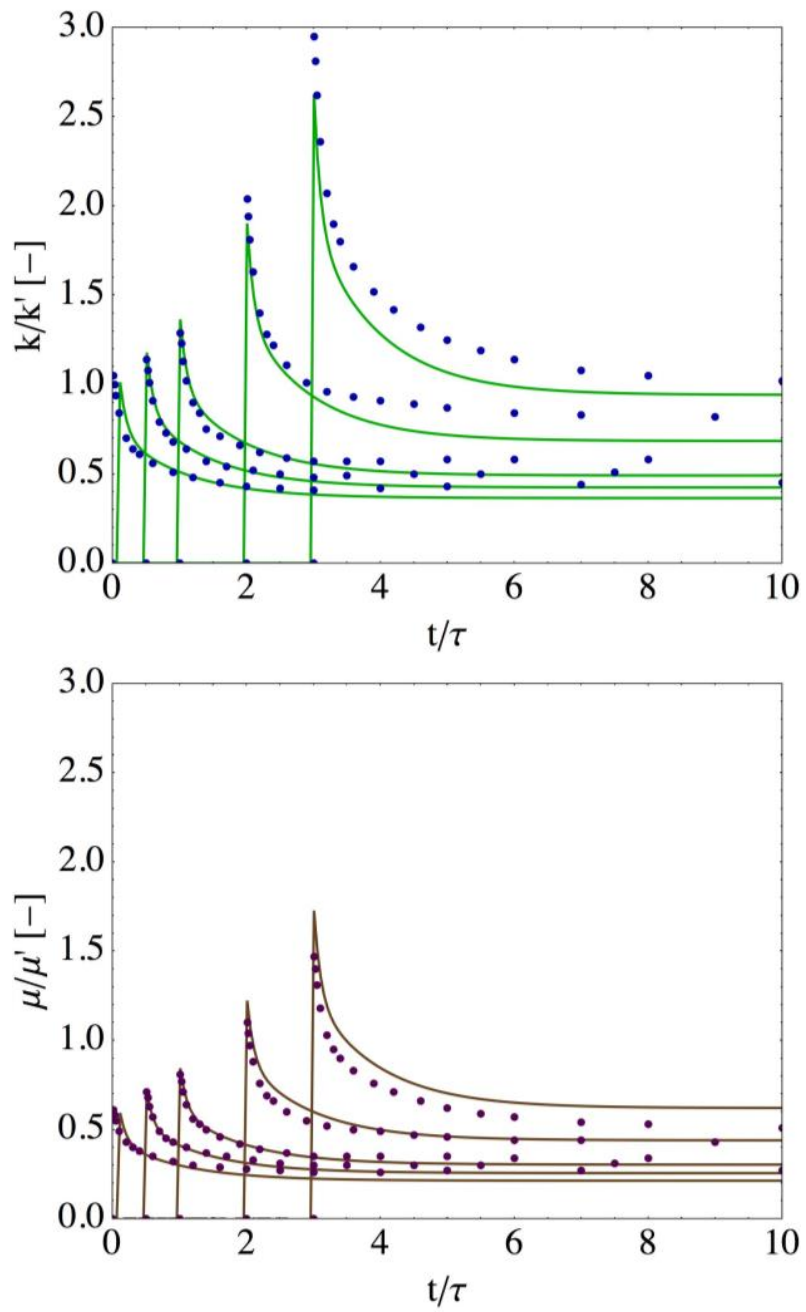

Figure 9: Bulk and shear relaxation functions: comparison between analytical (full lines) estimation of spherical inclusions and numerical simulations (dots) of polyhedral inclusions. We considered $40 \%$ of inclusions and the ageing function is linear $\left(f_{\text {age }}^{B}\right)$.

We highlight the following points:

- Discretization of the solidification process in the range studied here plays a significant role in analytical estimations but not in numerical simulations.

- The boundary conditions does not influence significantly the results in the cases studied.

- The shape of inclusions considered in 
this study seems to play a minor role in the estimations. This adds confidence in the use of the analytical estimations (based on spherical inclusions) to the study of composites with non-spherical (but with aspect ratio relatively close to 1) inclusions. Further investigations are, though, necessary to verify this effect in composites with higher content of inclusions and/or with flattened or elongated inclusions with aspect ratio significantly different from 1 .

Other perspectives of this work include accounting for intrinsically ageing behaviors associated to the matrix or inclusions, as can be found in cement based materials due for example to space-filling processes $[18,19]$. Similar combined analytical and numerical studies can be performed to investigate the thermal properties [20], notably the thermal expansions, of composites presenting an ageing viscoelastic behavior.

\section{REFERENCES}

[1] T. Honorio, B. Bary, J. Sanahuja, F. Benboudjema, Effective properties of n-coated composite spheres assemblage in an ageing viscoelastic framework, (submitted).

[2] J. Sanahuja, Effective behaviour of ageing linear viscoelastic composites: Homogenization approach, Int. J. Solids Struct. 50 (2013) 28462856.

[3] Z.P. Bazant, Viscoelasticity of Solidifying Porous Material - Concrete, J. Eng. Mech. Div. 103 (1977) 1049-1067.

[4] J. Sanahuja, Efficient Homogenization of Ageing Creep of Random Media: Application to Solidifying Cementitious Materials, in: American Society of Civil Engineers, 2013: pp. 201-210.

[5] B. Bary, L. Gélébart, E. Adam, C. Bourcier, Numerical analysis of linear viscoelastic 3D concrete specimens: Comparison between FE and FFT methods, in: Comput. Model. Concr. Struct., St. Anton am Arlberg, Austria, 2014: pp. 373-381.

[6] C. Bourcier, W. Dridi, L. Chomat, E. Laucoin, B. Bary, E. Adam, Combs: open source python library for RVE generation. Application to microscale diffusion simulations in cementitious materials, in: D. Caruge, C. Calvin, C.M. Diop, F. Malvagi, J.-C. Trama (Eds.), EDP Sciences, 2014: p. 02107.

[7] Z.P. Bazant, Viscoelasticity of Solidifying Porous
Material ${ }^{\circ}$ Concrete, J. Eng. Mech. Div. 103 (1977) 1049-1067.

[8] I. Carol, Z. Bažant, Viscoelasticity with Aging Caused by Solidification of Nonaging Constituent, J. Eng. Mech. 119 (1993) 2252-2269.

[9] Sanahuja, Julien, M. Di Ciaccio, From Micromechanisms to Mechanical Behaviour: An Application to the Ageing Creep of a Cement Paste, in: CONCREEP 10, American Society of Civil Engineers, n.d.: pp. 1024-1027.

[10] J. Sanahuja, Homogenization of Solidifying Random Porous Media: Application to Ageing Creep of Cementitious Materials, in: Beijing, China, 2014.

[11] J. Salençon, Viscoélasticité pour le calcul des structures, Editions Ecole Polytechnique, 2009.

[12] B. Bary, L. Gélébart, E. Adam, C. Bourcier, Numerical analysis of linear viscoelastic 3D concrete specimens, in: Comput. Model. Concr. Struct., CRC Press, 2014: pp. 373-381.

[13] C. Bourcier, W. Dridi, L. Chomat, E. Laucoin, B. Bary, E. Adam, Combs: open source python library for RVE generation. Application to microscale diffusion simulations in cementitious materials, in: D. Caruge, C. Calvin, C.M. Diop, F. Malvagi, J.-C. Trama (Eds.), EDP Sciences, 2014: p. 02107.

[14] B. Bary, M.B. Haha, E. Adam, P. Montarnal, Numerical and analytical effective elastic properties of degraded cement pastes, Cem. Concr. Res. 39 (2009) 902-912.

[15] S. Pecullan, L.V. Gibiansky, S. Torquato, Scale effects on the elastic behavior of periodic andhierarchical two-dimensional composites, J. Mech. Phys. Solids. 47 (1999) 1509-1542.

[16] Z.P. Bažant, Numerical determination of longrange stress history from strain history in concrete, Matér. Constr. 5 (1972) 135-141.

[17] T. Helfer, B. Michel, J.-M. Proix, M. Salvo, J. Sercombe, M. Casella, Introducing the opensource mfront code generator: Application to mechanical behaviours and material knowledge management within the PLEIADES fuel element modelling platform, Comput. Math. Appl. 70 (2015) 994-1023.

[18] T. Honorio, Modelling concrete behaviour at earlyage: multiscale analysis and simulation of a massive disposal structure, $\mathrm{PhD}$ Thesis, ENS Cachan (Univeristé Paris-Saclay), 2015.

[19] T. Honorio, B. Bary, F. Benboudjema, Multiscale estimation of the viscoelastic properties of cementbased materials at early age: a combined analytical and numerical approach, (submitted).

[20] T. Honorio, B. Bary, F. Benboudjema, Multiscale estimation of the thermal properties of cementbased materials at early-age, in: SSCS 2015 Numer. Model. - Strateg. Sustain. Concr. Struct., Rio de Janeiro, Brazil, 2015. 